\title{
Estimativa da Radiação Solar Global com Base em Observações de Temperatura para o Estado de Goiás
}

\author{
Diego Simões Fernandes ${ }^{1,2}$ iD , Alexandre Bryan Heinemann ${ }^{3}$ iD , André de Oliveira Amorim², \\ Rosidalva Lopes Feitosa da $\mathrm{Paz}^{2}$ \\ ${ }^{1}$ Universidade Estadual de Goiás, Câmpus Palmeiras de Goiás, \\ Palmeiras de Goiás, GO, Brasil. \\ ${ }^{2}$ Instituto Estadual de Meteorologia e Tecnologias Sociais de Goiás, Goiânia, GO, Brasil. \\ ${ }^{3}$ Centro Nacional de Pesquisa em Arroz e Feijão, \\ Empresa Brasileira de Pesquisa Agropecuária, Santo Antônio de Goiás, GO, Brasil.
}

Recebido em 7 de Junho de 2016 - Aceito em 18 de Julho de 2018

\begin{abstract}
Resumo
O objetivo deste trabalho foi avaliar o desempenho de cinco modelos de estimativa de radiação solar baseados em dados de temperatura para os períodos seco e úmido no estado de Goiás. Dados climáticos de 10 municípios foram utilizados. O desempenho dos modelos na calibração e validação foi avaliado por meio do coeficiente de determinação $\left(\mathrm{R}^{2}\right)$, erro quadrático médio (EQM), erro relativo quadrático médio (ERQM), erro médio absoluto (EMA) e pela eficiência do modelo por meio do método de Nash-Sutcliff (EF). Além disso, também foi avaliado o desempenho dos modelos considerando toda a série histórica dos dados estimados de radiação solar diária e mensal por meio dos índices de concordância de Willmott, de confiança de Camargo, função "spline" de alisamento cúbica e regressão linear. Observou-se que os modelos HG e DCBB apresentaram os piores e os modelos CD e DB os melhores desempenhos para estimar a radiação solar para o estado de Goiás.
\end{abstract}

Palavras-chave: Bristow-Campbell, Campbell-Donatelli, Donatelli-Bellocchi, Hargreaves.

\section{Estimation of Global Solar Radiation Based in Temperature Observations for the Goiás State}

\begin{abstract}
The objective of this work was to evaluate the performance of five models for estimating solar radiation based on temperature data for dry and wet periods in Goiás State. Climate data from 10 municipalities were used. The models performance for calibration and validation were evaluated by coefficient of determination $\left(\mathrm{R}^{2}\right)$, root mean square error (RMSE), mean square relative error (RRMSE), the mean absolute error (MAE) and efficiency of model by the Nash-Sutcliff method (EF). Also, the models performance considering all data set for daily and monthly estimated solar radiation were evaluated by Willmott's agreement coefficient, Camargo's confidence index, spline smoothing cubic regression and linear regression. It was observed that HG and DCBB models showed the worst performance and CD and DB models the best performance for estimating solar radiation values for Goiás State.
\end{abstract}

Keywords: Bristow-Campbell, Campbell-Donatelli, Donatelli-Bellocchi, Hargreaves.

\section{Introdução}

O estado de Goiás se destaca por sua vocação agrícola, principalmente na produção brasileira de soja e milho primeira safra. Para a sustentabilidade desse sistema agrícola, e devido a variabilidade e possíveis mudanças climá- ticas, há um grande interesse em conhecer as interações entre clima e genótipos (Cargnin et al., 2006; Cruz e Regazzi, 1997). Vários pesquisadores (Mavromatis e Jagtap, 2005; Abraha e Savage, 2008; Bansouleh et al., 2009) têm demonstrado que modelos de simulação da produtividade, crescimento e desenvolvimento de culturas são ferramentas

Autor de correspondência: Diego Simões Fernandes, diego.fernandes@ueg.br. 
úteis para avaliar os impactos do clima sobre a agricultura. Outros trabalhos apontam que esses modelos podem ser utilizados para a estimativa da produtividade anual (Jagtap e Jones, 2002), para adequar o manejo da cultura às condições climatológicas (Mavromatis et al., 2002) e suporte a programas de melhoramento vegetal (Heinemann et al., 2009; Heinemann, 2010). Entretanto, a aplicação desses modelos depende da disponibilidade das seguintes variáveis: características de solo, coeficientes dos genótipos da cultura e séries históricas climáticas, composta por dados diários de precipitação, temperatura máxima e mínima e radiação solar global. Dentre essas variáveis, para o estado de Goiás, a maior limitação para a aplicação dessas ferramentas é a disponibilidade de séries históricas diárias de radiação solar global (Heinemann et al., 2008).

A radiação solar é um dos principais fatores climáticos que interferem de forma significativa na produção agrícola, pois a quantidade de radiação recebida pelas plantas irá encadear processos fundamentais para a elaboração de assimilados resultantes da fotossíntese (Tei et al., 1996). Também, é de grande importância para o ciclo hidrológico, pois é a fonte fundamental de energia do ciclo na biosfera, exercendo influência nas possibilidades agrícolas de cada região por meio das características de distribuição das chuvas, que por sua vez está sujeita, nas zonas tropicais, do movimento do sol e da distribuição da radiação (Tundisi, 2003). Na ausência de observações coletadas em estações meteorológicas de radiação solar global, existem alternativas para estimar a radiação solar diária: por meio de imagens de satélite (Pinker et al., 1995; Ceballos, 2000), geração estocástica (Hansen, 1999) e modelos empíricos (Liu e Scott, 2001; Mahmood e Hubbard, 2002). Devido à complexidade de estimar a radiação solar por meio de imagens de satélites, e a não disponibilidade de séries históricas diárias de radiação solar para a aplicação de modelos estocásticos, esse estudo objetiva a avaliação de modelos empíricos de estimativa de radiação solar global associada a observações de temperatura máxima e mínima para o estado de Goiás. Os modelos empíricos de estimativa da radiação solar global utilizados nesse estudo foram: Hargreaves (HG, Hargreaves et al., 1985), Bristow-Campbell (BC, Bristow e Campbell, 1984), Campbell-Donatelli (CD, Donatelli e Campbell, 1998), Donatelli-Bellocchi (DB, Donatelli e Bellocchi, 2001) e modelo modular DCBB (DCBB, Donatelli et al., 2003).

\section{Material e Métodos}

A região de estudo compreende o estado de Goiás, localizado entre os meridianos $45^{\circ}$ e $54^{\circ} \mathrm{O}$ e os paralelos $12^{\circ}$ e $20^{\circ} \mathrm{S}$. Essa região apresenta dois períodos bem definidos, seco (abril- setembro) e úmido (outubro-março), e sofre influência dos sistemas meteorológicos de origem tropical (Complexo Convectivos de Mesoescala) os quais atuam mais ao norte da região proveniente da região amazônica, e extratropical (frentes frias e linhas de instabi- lidade associadas a sistemas frontais) mais ao sul da região (Reboita et al., 2010). As estações meteorológicas na qual os dados meteorológicos foram utilizados pertencem à rede de observação do Instituto Estadual de Meteorologia e Tecnologias Sociais de Goiás (IEMETES) e a disposição espacial das mesmas está ilustrada na Fig. 1. As variáveis meteorológicas diárias utilizadas foram temperatura máxima e mínima do ar e radiação solar global. A consistência e qualidade dessas variáveis foram verificadas utilizando-se a metodologia descrita por Heinemann et al. (2007). O número de anos, período, localização geográfica e municípios que essas estações pertencem estão descritos na Tabela 1.

Nesse estudo, a radiação solar global diária foi obtida como o produto da transmissividade da radiação solar atmosférica e da radiação potencial (extraterrestre). A radiação potencial $\left(R_{a}\right)$ foi expressa quantitativamente em função da latitude e dia do ano. A transmissividade em céu claro foi fixado em 0,75 conforme sugerido por Weiss et al. (2001) e Fletcher e Moot (2007). As Eqs. (1)-(5) ilustram os modelos HG, BC, CD, DB e DCBB utilizados para estimar a radiação solar global diária.

$\operatorname{Rad}_{\mathrm{HG}}=b R_{a} \sqrt{T_{\text {max }}-T_{\text {min }}}+c$

$\operatorname{Rad}_{\mathrm{BC}}=\left[1-\exp \left(\frac{-b \Delta T_{i}^{c}}{\Delta T_{\text {avg }}}\right)\right] \cdot R a$

$\operatorname{Rad}_{\mathrm{CD}}=0,75\left[1-\exp \left(-b\left(0,017 \exp \left(\exp \left(-0,053 \Delta T_{\text {avg }}\right)\right)\right)\right] \cdot \operatorname{Ra}(3)\right.$

$\operatorname{Rad}_{\mathrm{DB}}=0,75\left[1-f_{2}(i)\right]\left[1-\exp \left(\frac{-b \Delta T_{i}^{2}}{\Delta T_{a v g}}\right)\right] \cdot R a$

$\operatorname{Rad}_{\mathrm{DCBB}}=0,75\left[1-f_{2}(i)\right]\left[\frac{-b \Delta T_{i}^{2} f 1(T \text { min })}{\Delta T_{\text {avg }}}\right] \cdot R a$

sendo $\Delta T=\operatorname{Tmax}_{i}-\left(\operatorname{Tmin}_{i}+\operatorname{Tmin}_{i+1}\right) / 2\left(\operatorname{com}_{i}\right.$ representado o dia), $\Delta T_{\text {avg }}$ média móvel centrada para 7 dias, $f 1($ Tmin $)=$ $\exp \left(\operatorname{Tmin}_{i} / \operatorname{Tnc}\right), f 2(i)=C 1[\operatorname{sen}(i \times C 2 \times \pi / 180)+\cos (i \times$ $f 3(C 2) \times \pi / 180)$ ], sendo Tmax e Tmin as temperaturas máxima e mínima diária do $\operatorname{ar}\left({ }^{\circ} \mathrm{C}\right), f 3(C 2)=1-1,9 \times C 3+$ $3,83 \times C 3^{2}$, sendo $C 3=C 2$ - inteiro $(C 2)$. Tnc $, b, c, C 1$ e $C 2$ são parâmetros empíricos dos modelos.

Dentre esses modelos, o HG se destaca por estimar a radiação solar global de maneira simples relacionando está a amplitude térmica diária (Trnka et al., 2005). O modelo $\mathrm{BC}$ explora a relação entre a amplitude térmica diária e a irradiância para estimar o fluxo diário da radiação solar que chega à superfície. Alguns estudos tem desenvolvido melhorias neste modelo (Goodin et al., 1999, McVicar e Jupp, 1999). Nesse estudo, para a correção da amplitude termica $(\Delta T)$, no modelo $\mathrm{BC}$, foi utilizado a média movel centrada $\left(\Delta T_{\text {avg }}\right)$. O modelo CD considera situações na qual a temperatura do ar a noite apresenta um resfriamento menor que a 


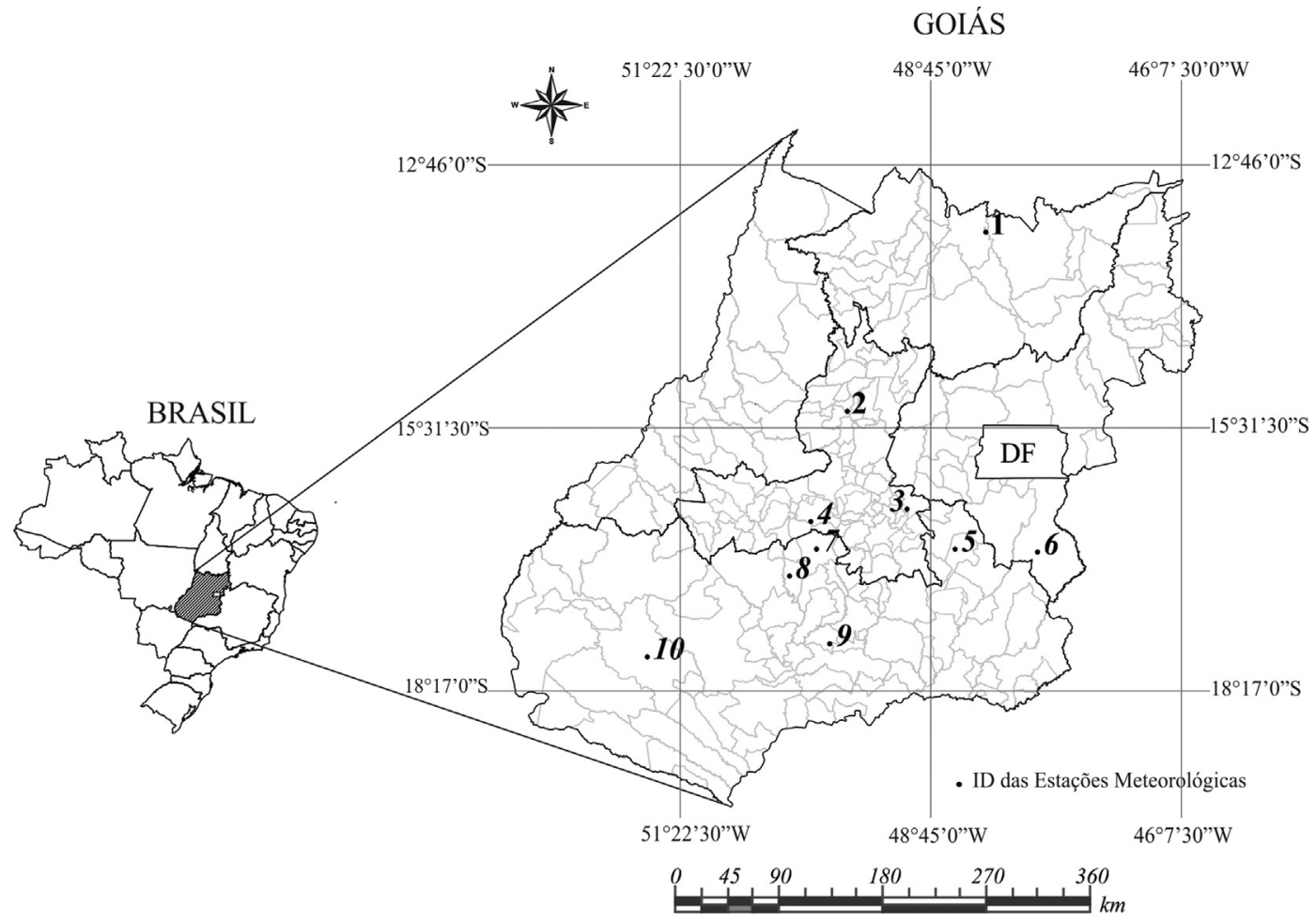

Figura 1 - Mapa com a localização geográfica das estações meteorológicas utilizadas no estudo. Números representam as IDs das estações meteorológicas conforme descritos na Tabela 1.

Tabela 1 - Localização geográfica, altitude, período e número de anos utilizados das estações meteorológicas avaliadas nesse estudo.

\begin{tabular}{lcccccc}
\hline ID & Município & Lat $\left(^{\circ}\right)$ & Lon $\left(^{\circ}\right)$ & Alt $(\mathrm{m})$ & Período & $\mathrm{N}^{\circ}$ de anos \\
\hline 01 & Minaçu & 13,40 & 48,05 & 343 & $2004-2009$ & 6 \\
02 & Ceres & 15,31 & 49,60 & 739 & $2002-2007$ & 6 \\
03 & Anápolis & 16,33 & 48,95 & 1136 & $2005-2008$ & 4 \\
04 & Anicuns & 16,46 & 49,96 & 692 & $2006-2009$ & 4 \\
05 & Vianópolis & 16,74 & 48,52 & 1110 & $2004-2007$ & 4 \\
06 & Cristalina & 16,77 & 47,61 & 1189 & $2005-2008$ & 4 \\
07 & Palmeiras de Goiás & 16,80 & 49,93 & 596 & $2000-2001$ e 2004-2005 & 4 \\
08 & Jandaia & 17,05 & 50,15 & 637 & $2005-2008$ & 4 \\
09 & Vicentinópolis & 17,74 & 49,81 & 648 & $2000-2005$ & 6 \\
10 & Jataí & 17,88 & 51,71 & 696 & $2004-2009$ & 6 \\
\hline
\end{tabular}

ID - identidade; Lat - latitude; Lon - longitude; Alt - altitude.

temperatura do ar durante o dia (Mavromatis e Jagtap, 2005). Diferente dos modelos anteriores, o modelo DB leva em consideração o efeito da variação sazonal da transmissividade e da variação da temperatura utilizando funções trigonométricas. O modelo DCBB inclui as caracteristicas dos modelos $\mathrm{BC}, \mathrm{CD}$ e $\mathrm{DB}$, permitindo ativar ou não essas funções, por meio de equações trigonométricas.

A calibração dos parâmetros empíricos dos modelos de estimativa de radiação solar global diária baseou-se nas condições climáticas de cada uma das estações meteorológicas descritas na Tabela 1. Para a calibração desses parâmetros utilizou-se somente os anos ímpares, e para a validação, somente os anos pares. Essa metodologia reduz o efeito de erros que podem ocorrer de um ano para o outro. Os parâmetros empíricos dos modelos $\mathrm{BC}, \mathrm{CD}, \mathrm{DB}$ e DCBB foram ajustados pela minimização do erro quadrático médio. Já, os parâmetros empíricos referente ao modelo HG foram ajustados pelo método de iteração, por meio do ajuste de modelos não lineares da função nls (non-linear least squares) do pacote MASS do software R (Venables e Ripley, 2002).

A acurácia dos modelos de estimativa de radiação solar para a calibração e validação foi avaliada utilizando-se o coeficiente de determinação $\left(\mathrm{R}^{2}\right)$, o erro quadrático médio (EQM), o erro relativo quadrático médio (ERQM), o erro 
médio absoluto (EMA) e a eficiência (EF) do modelo pelo método de Nash-Sutcliff (Nash e Sutcliff, 1970).

Os valores diários e os acumulados mensais da radiação solar estimada pelos cinco diferentes modelos foram divididos nos períodos seco (Abril a Setembro) e úmido (Outubro a Maio) e a acurácia dos modelos nesses períodos avaliadas por meio do índice de concordância de Willmott (Willmott e Wicks, 1980), índice de confiança de Camargo (Camargo e Sentelhas, 1997) e da função "spline" de alisamento cúbica (cubic smoothing spline, Ramsay et al., 1997).

\section{Resultados e Discussão}

Os modelos de estimativa de radiação solar global utilizados aqui são baseados nos valores extremos da temperatura do ar diária. A variação da amplitude térmica na região de estudo está ilustrada pela Fig. 2. As menores amplitudes térmicas ocorrem nos meses úmidos (Out, Nov, Dez, Jan, Fev e Mar). Já, os meses pertencentes ao período seco apresentaram as maiores amplitudes térmicas. Isso ocorre porque nesse período, à noite, não há presença de nuvens, favorecendo a perda radiativa e ocasionando maiores amplitudes térmicas (Froelich e Schmid, 2006).

A Tabela 2 descreve os valores dos parâmetros empíricos calibrados para as 10 localidades (Fig. 1). O parâmetro "b" para os modelos HG, BC, CD, DB e DCBB, variou de $0,13(\mathrm{CD})$ a 1,93 (BC). Valiati (2005) encontrou valores semelhantes para o parâmetro "b" para os modelos BC $(0,136), \mathrm{CD}(0,477), \mathrm{DB}(0,144)$ e $\mathrm{DCBB}(0,112)$ no município de Botucatu-SP. O modelo BC apresentou os maiores valores para o parâmetro "b". Isso ocorre porque esse parâmetro é afetado pela forma na qual a amplitude é calculada (Liu et al., 2008) e nesse estudo, como explicado na metodologia, foi utilizado como fator de correção da
$\Delta$ Ta média móvel centrada $\left(\Delta \mathrm{T}_{\text {avg }}\right)$. O parâmetro "c", para os modelos $\mathrm{HG}$ e $\mathrm{BC}$, variou de $-4,8$ a 1,89. Os valores dos coeficientes $\mathrm{c} 1$ e $\mathrm{c} 2$ foram similares para os modelos DB e DCBB e variaram de $-0,06$ a 0,06 e 0,46 a 1,46 , respectivamente. De acordo com os autores Bristow e Campbell (1984), Donatelli e Campbell (1998) e Donatelli e Belochi (2001), quanto mais próximos esses coeficientes de zero, menor é a amplitude térmica. Isso indica que há variação sazonal em relação à temperatura do ar nessa região. $\mathrm{O}$ parâmetro Tnc variou de 16,09 (CD) a 200 (DCBB).

Os índices de desempenho (ERQM, EQM, EMA, EF e $\mathrm{R}^{2}$ ) para calibração e validação dos modelos para as diferentes localidades estão descritos na Tabela 3. Como

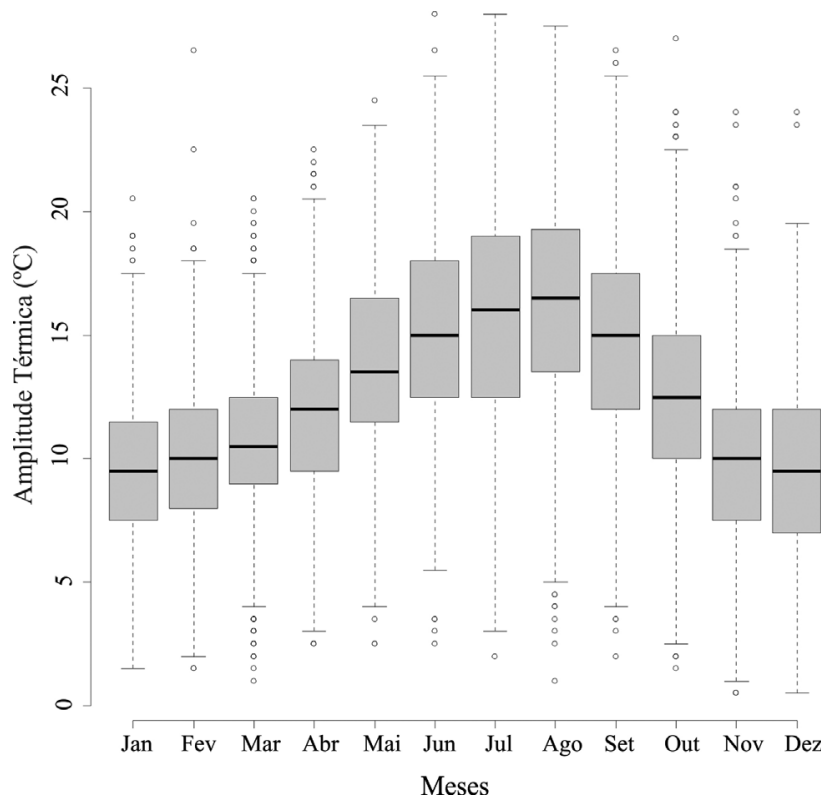

Figura 2 - Amplitude térmica mensal considerando dados de temperatura de todas as 10 localidades.

Tabela 2 - Valores dos parâmetros empíricos b, c, $\mathrm{c}_{1}, \mathrm{c}_{2}$, Tnc, utilizados para o processo de calibração diária dos modelos.

\begin{tabular}{|c|c|c|c|c|c|c|c|c|c|c|c|}
\hline \multirow[t]{2}{*}{ Modelo } & \multirow[t]{2}{*}{ Parâmetro } & \multicolumn{10}{|c|}{ ID (Município) } \\
\hline & & 1 & 2 & 3 & 4 & 5 & 6 & 7 & 8 & 9 & 10 \\
\hline \multirow[t]{2}{*}{ HG } & $\mathrm{b}$ & 0,19 & 0,18 & 0,17 & 0,18 & 0,18 & 0,16 & 0,17 & 0,17 & 0,14 & 0,18 \\
\hline & $\mathrm{c}$ & $-2,67$ & $-4,80$ & $-0,07$ & $-1,98$ & $-3,69$ & 1,42 & $-4,03$ & $-2,15$ & 0,82 & $-2,71$ \\
\hline \multirow[t]{2}{*}{$\mathrm{BC}$} & $\mathrm{b}$ & 0,61 & 0,96 & 0,70 & 0,75 & 0,69 & 0,39 & 0,87 & 1,93 & 0,42 & 0,94 \\
\hline & $\mathrm{c}$ & 1,70 & 1,38 & 1,60 & 1,59 & 1,47 & 1,89 & 1,36 & 1,12 & 1,71 & 1,42 \\
\hline \multirow[t]{2}{*}{$\mathrm{CD}$} & $\mathrm{b}$ & 0,44 & 0,13 & 0,46 & 0,42 & 0,18 & 0,51 & 0,13 & 0,13 & 0,30 & 0,23 \\
\hline & Tnc & 110,00 & 19,59 & 110,00 & 110,00 & 26,28 & 110,00 & 23,68 & 16,09 & 110,00 & 28,62 \\
\hline \multirow[t]{3}{*}{ DB } & $\mathrm{b}$ & 0,30 & 0,20 & 0,28 & 0,28 & 0,19 & 0,30 & 0,17 & 0,24 & 0,21 & 0,24 \\
\hline & $\mathrm{c} 1$ & $-0,02$ & 0,04 & $-0,02$ & 0,00 & 0,05 & $-0,06$ & 0,04 & 0,06 & 0,02 & 0,01 \\
\hline & c2 & 1,42 & 1,41 & 1,46 & 1,22 & 0,46 & 1,46 & 0,76 & 1,03 & 0,74 & 0,74 \\
\hline \multirow[t]{4}{*}{ DCBB } & $\mathrm{b}$ & 0,25 & 0,17 & 0,24 & 0,23 & 0,16 & 0,26 & 0,14 & 0,20 & 0,17 & 0,20 \\
\hline & $\mathrm{c} 1$ & -0.03 & 0.04 & -0.02 & 0 & 0.04 & -0.06 & 0.04 & 0.05 & 0.01 & 0.01 \\
\hline & $\mathrm{c} 2$ & 1,42 & 1,41 & 1,46 & 1,22 & 0,46 & 1,46 & 0,76 & 1,03 & 0,74 & 0,74 \\
\hline & Tnc & 140,05 & 41,77 & 84,10 & 107,55 & 44,23 & 78,22 & 33,68 & 41,58 & 200,00 & 44,37 \\
\hline
\end{tabular}

b, c, c c $1, c_{2}$ e Tnc - parâmetros; BC - Bristow-Campbell; CD - Campbell-Donatelli; DB - Donatelli-Bellocchi; DCBB - modelo Modular; HG - Hargreaves. 
Tabela 3 - Índices de desempenho dos modelos BC - Bristow-Campbell; CD - Campbell-Donatelli; DB - Donatelli-Bellocchi; DCBB - modelo Modular; HG - Hargreaves; EQM - erro quadrático médio; ERQM - erro relativo quadrático médio; EMA - erro médio absoluto; EF - eficiência do modelo; R² coeficiente de determinação.

\begin{tabular}{|c|c|c|c|c|c|c|c|c|c|c|c|}
\hline \multirow[t]{2}{*}{ Modelo } & \multirow[t]{2}{*}{ ID } & \multicolumn{5}{|c|}{ Calibração } & \multicolumn{5}{|c|}{ Validação } \\
\hline & & EQM & ERQM & EMA & $\mathrm{EF}$ & $\mathrm{R}^{2}$ & EQM & ERQM & EMA & $\mathrm{EF}$ & $\mathrm{R}^{2}$ \\
\hline \multirow[t]{11}{*}{$\mathrm{HG}$} & 1 & 3,79 & 13,83 & 2,98 & 0,53 & 0,53 & 4,15 & 15,12 & 3,29 & 0,41 & 0,43 \\
\hline & 2 & 3,16 & 11,82 & 2,43 & 0,59 & 0,59 & 3,35 & 12,52 & 2,56 & 0,53 & 0,54 \\
\hline & 3 & 3,90 & 13,14 & 3,07 & 0,39 & 0,39 & 3,91 & 16,12 & 3,15 & 0,39 & 0,39 \\
\hline & 4 & 3,73 & 13,24 & 2,91 & 0,49 & 0,49 & 3,79 & 13,47 & 2,99 & 0,42 & 0,43 \\
\hline & 5 & 4,40 & 16,65 & 3,37 & 0,46 & 0,46 & 4,40 & 16,65 & 3,48 & 0,38 & 0,44 \\
\hline & 6 & 4,35 & 14,88 & 3,54 & 0,34 & 0,34 & 4,04 & 16,91 & 3,34 & 0,37 & 0,37 \\
\hline & 7 & 2,66 & 10,30 & 2,10 & 0,67 & 0,66 & 3,24 & 12,53 & 2,57 & 0,56 & 0,57 \\
\hline & 8 & 3,27 & 11,51 & 2,46 & 0,52 & 0,52 & 3,14 & 13,50 & 2,39 & 0,54 & 0,55 \\
\hline & 9 & 3,64 & 15,30 & 2,84 & 0,39 & 0,39 & 3,76 & 15,77 & 3,04 & 0,43 & 0,43 \\
\hline & 10 & 3,57 & 13,58 & 2,73 & 0,57 & 0,57 & 3,63 & 13,78 & 2,84 & 0,52 & 0,51 \\
\hline & Média & 3,69 & 13,41 & 2.88 & 0,51 & 0.51 & 3,78 & 14,45 & 3,02 & 0,43 & 0,44 \\
\hline \multirow[t]{11}{*}{$\mathrm{BC}$} & 1 & 3,18 & 11,59 & 2,38 & 0,67 & 0,67 & 3,73 & 13,57 & 2,84 & 0,53 & 0,55 \\
\hline & 2 & 3,10 & 11,58 & 2,30 & 0,61 & 0,61 & 3,15 & 11,76 & 2,35 & 0,59 & 0,59 \\
\hline & 3 & 3,65 & 12,31 & 2,73 & 0,46 & 0,47 & 3,62 & 14,93 & 2,79 & 0,48 & 0,49 \\
\hline & 4 & 3,55 & 12,62 & 2,69 & 0,54 & 0,55 & 3,65 & 12,95 & 2,80 & 0,47 & 0,47 \\
\hline & 5 & 4,25 & 16,09 & 3,08 & 0,50 & 0,50 & 4,16 & 15,72 & 3,06 & 0,45 & 0,50 \\
\hline & 6 & 3,82 & 13,06 & 2,93 & 0,49 & 0,52 & 3,62 & 15,16 & 2,83 & 0,50 & 0,54 \\
\hline & 7 & 2,56 & 9,92 & 2,05 & 0,69 & 0,69 & 2,97 & 11,48 & 2,24 & 0,63 & 0,65 \\
\hline & 8 & 3,24 & 11,41 & 2,38 & 0,53 & 0,53 & 3,09 & 13,28 & 2,31 & 0,55 & 0,57 \\
\hline & 9 & 3,15 & 13,25 & 2,43 & 0,54 & 0,55 & 3,56 & 14,94 & 2,85 & 0,49 & 0,51 \\
\hline & 10 & 3,44 & 13,09 & 2,57 & 0,60 & 0,60 & 3,54 & 13,45 & 2,66 & 0,54 & 0,54 \\
\hline & Média & 3,34 & 12,47 & 2,50 & 0,54 & 0,55 & 3,59 & 13,51 & 2,80 & 0,52 & 0,54 \\
\hline \multirow[t]{11}{*}{$\mathrm{CD}$} & 1 & 3,30 & 12,04 & 2,46 & 0,64 & 0,68 & 3,81 & 13,88 & 2,86 & 0,50 & 0,56 \\
\hline & 2 & 3,23 & 12,06 & 2,37 & 0,57 & 0,62 & 3,25 & 12,14 & 2,42 & 0,56 & 0,59 \\
\hline & 3 & 3,77 & 12,72 & 2,76 & 0,43 & 0,48 & 3,78 & 15,61 & 2,84 & 0,43 & 0,50 \\
\hline & 4 & 3,67 & 13,05 & 2,71 & 0,51 & 0,55 & 3,77 & 13,40 & 2,80 & 0,43 & 0,48 \\
\hline & 5 & 4,28 & 16,18 & 3,10 & 0,49 & 0,52 & 4,24 & 16,03 & 3,03 & 0,42 & 0,49 \\
\hline & 6 & 3,89 & 13,30 & 2,99 & 0,47 & 0,52 & 3,71 & 15,53 & 2,91 & 0,47 & 0,54 \\
\hline & 7 & 2,66 & 10,27 & 2,06 & 0,67 & 0,70 & 2,90 & 11,19 & 2,16 & 0,65 & 0,66 \\
\hline & 8 & 3,55 & 12,49 & 2,59 & 0,43 & 0,54 & 3,34 & 14,38 & 2,43 & 0,47 & 0,57 \\
\hline & 9 & 3,24 & 13,64 & 2,50 & 0,51 & 0,56 & 3,59 & 15,10 & 2,88 & 0,47 & 0,53 \\
\hline & 10 & 3,57 & 13,59 & 2,64 & 0,57 & 0,60 & 3,71 & 14,11 & 2,76 & 0,49 & 0,54 \\
\hline & Média & 3,56 & 12,89 & 2,62 & 0,51 & 0,56 & 3,71 & 14,25 & 2,82 & 0,47 & 0.54 \\
\hline \multirow[t]{11}{*}{ DB } & 1 & 3,29 & 12,00 & 2,45 & 0,64 & 0,68 & 3,82 & 13,90 & 2,85 & 0,50 & 0,57 \\
\hline & 2 & 3,43 & 12,82 & 2,54 & 0,52 & 0,59 & 3,37 & 12,58 & 2,58 & 0,53 & 0,56 \\
\hline & 3 & 3,77 & 12,72 & 2,73 & 0,43 & 0,48 & 3,75 & 15,49 & 2,80 & 0,44 & 0,50 \\
\hline & 4 & 3,66 & 13,00 & 2,68 & 0,51 & 0,55 & 3,73 & 13,24 & 2,77 & 0,44 & 0,48 \\
\hline & 5 & 4,37 & 16,53 & 3,09 & 0,47 & 0,51 & 4,49 & 16,98 & 3,25 & 0,35 & 0,44 \\
\hline & 6 & 3,81 & 13,04 & 2,85 & 0,49 & 0,53 & 3,71 & 15,53 & 2,88 & 0,47 & 0,54 \\
\hline & 7 & 3,12 & 12,07 & 2,53 & 0,54 & 0,64 & 3,09 & 11,92 & 2,31 & 0,60 & 0,63 \\
\hline & 8 & 3,37 & 11,85 & 2,45 & 0,49 & 0,57 & 3,29 & 14,15 & 2,40 & 0,49 & 0,57 \\
\hline & 9 & 3,23 & 13,58 & 2,49 & 0,52 & 0,56 & 3,67 & 15,41 & 2,96 & 0,45 & 0,50 \\
\hline & 10 & 3,67 & 13,96 & 2,71 & 0,55 & 0,59 & 3,81 & 14,47 & 2,81 & 0,47 & 0,52 \\
\hline & Média & 3,55 & 12,91 & 2,61 & 0,52 & 0,57 & 3,72 & 14,31 & 2,81 & 0,47 & 0,53 \\
\hline \multirow[t]{11}{*}{ DCBB } & 1 & 3,34 & 12,17 & 2,51 & 0,63 & 0,68 & 3,88 & 14,12 & 2,93 & 0,49 & 0,57 \\
\hline & 2 & 3,90 & 14,56 & 2,98 & 0,38 & 0,59 & 3,94 & 14,72 & 3,00 & 0,35 & 0,54 \\
\hline & 3 & 3,78 & 12,75 & 2,73 & 0,42 & 0,48 & 3,76 & 15,51 & 2,79 & 0,44 & 0,50 \\
\hline & 4 & 3,66 & 12,99 & 2,70 & 0,51 & 0,56 & 3,72 & 13,22 & 2,78 & 0,44 & 0,48 \\
\hline & 5 & 4,64 & 17,56 & 3,20 & 0,40 & 0,49 & 4,50 & 17,02 & 3,28 & 0,35 & 0,41 \\
\hline & 6 & 3,84 & 13,15 & 2,84 & 0,48 & 0,52 & 3,67 & 15,38 & 2,86 & 0,48 & 0,54 \\
\hline & 7 & 4,06 & 15,69 & 3,33 & 0,22 & 0,69 & 4,20 & 16,21 & 3,23 & 0,26 & 0,63 \\
\hline & 8 & 3,90 & 13,73 & 2,93 & 0,31 & 0,60 & 3,90 & 16,80 & 2,92 & 0,28 & 0,58 \\
\hline & 9 & 3,35 & 14,07 & 2,58 & 0,48 & 0,56 & 3,85 & 16,18 & 3,10 & 0,40 & 0,50 \\
\hline & 10 & 3,99 & 15,18 & 2,96 & 0,47 & 0,58 & 4,15 & 15,77 & 3,05 & 0,37 & 0,52 \\
\hline & Média & 3,87 & 13,90 & 2,89 & 0,45 & 0,57 & 3,89 & 15,64 & 2,97 & 0,39 & 0,53 \\
\hline
\end{tabular}

ID - Identidade das estações meteorológicas e respectivos municípios conforme descrito na Tabela 1. 
esperado, há uma maior acurácia e precisão na calibração do que na validação. A eficiência média das estimativas realizadas para os dados das localidades analisadas, para a calibração, variou de 0,45 (DCBB) a 0,54 (BC), enquanto que para a validação variou de 0,39 (DCBB) a 0,52 (BC). $O$ modelo $\mathrm{BC}$ foi o que obteve a melhor eficiência média e o menor ERQM para ambos os processos calibração e validação. Abraha e Savage (2008) encontraram valores médios de ERQM que variaram de 14,34 a $20,28 \%$, para as localidades de Cortez e Davis, nos estados de Colorado e Califórnia, Estados Unidos. Os coeficientes de determinação médios das localidades variaram de $0,51(\mathrm{HG})$ a 0,57 (DB e DCBB) para calibração e de 0,44 (HG) a 0,54 (BC e $\mathrm{CD}$ ) para a validação. O modelo $\mathrm{HG}$ foi o que apresentou os piores coeficientes de determinação. O EQM médio na calibração, para as localidades, variou de 3,34 (BC) a 3,87 M.J.m ${ }^{-2} \cdot$ dia $^{-1}$ (DCBB). Mavromatis e Jagtap (2005) obtiveram valores de EQM entre 1,24 e 1,41 M.J.m ${ }^{-2} \cdot$ dia $^{-1}$ para a calibração dos modelos $\mathrm{BC}, \mathrm{CD}, \mathrm{DB}$ e o modular DCBB em diferentes localidades da Florida, Estados Unidos. Já, para o norte da Itália, Bechini et al. (2000) encon-
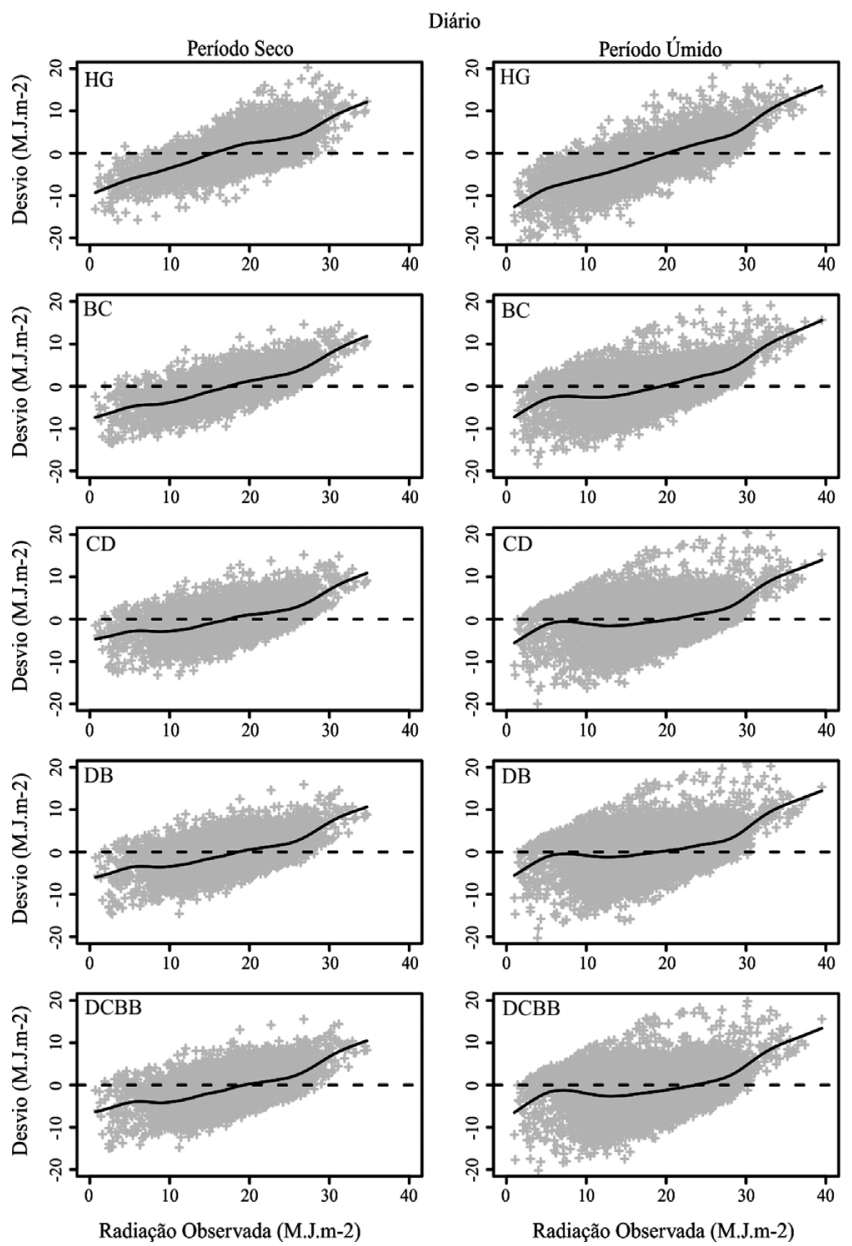

traram valores que variaram entre 2,3 e $3,9 \mathrm{M} . \mathrm{J} \cdot \mathrm{m}^{-2} \cdot \mathrm{dia}^{-1} \mathrm{e}$ Hunt et al. (1998), para o Canadá, obtiveram erros de 3,4 e 4,1 M.J.m. ${ }^{-2} \cdot \mathrm{dia}^{-1}$. O EMA teve a mesma tendência do EQM, sendo que o menor valor médio de EMA para as localidades foi observado para o modelo BC, tanto para a calibração e validação.

A Fig. 3 ilustra os desvios da radiação estimada pelos modelos tendo a função "spline" de alisamento cúbica ajustada em função dos valores observados diários e mensais de radiação para os períodos seco e úmido. Por meio dessa figura observou-se que para todos os modelos estudados, os maiores desvios ocorreram no período seco. Isso ocorre devido ao fato desse período apresentar menor variabilidade nos dados observados de radiação solar comparado ao período úmido, pois no período seco é muito pouco ou nenhuma o surgimento de nebulosidade que faz variar a radiação que chega à superfície. Para o período seco, dados diários, o modelo HG subestimou os valores de radiação solar global menores que 15 M.J.m ${ }^{-2} \cdot \mathrm{dia}^{-1}$ e superestimou acima desse valor. Enquanto que para o período úmido, esse mesmo modelo subestimou para valores abaixo de

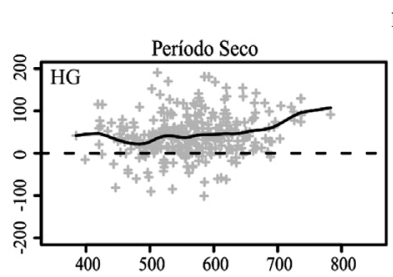

Mensal
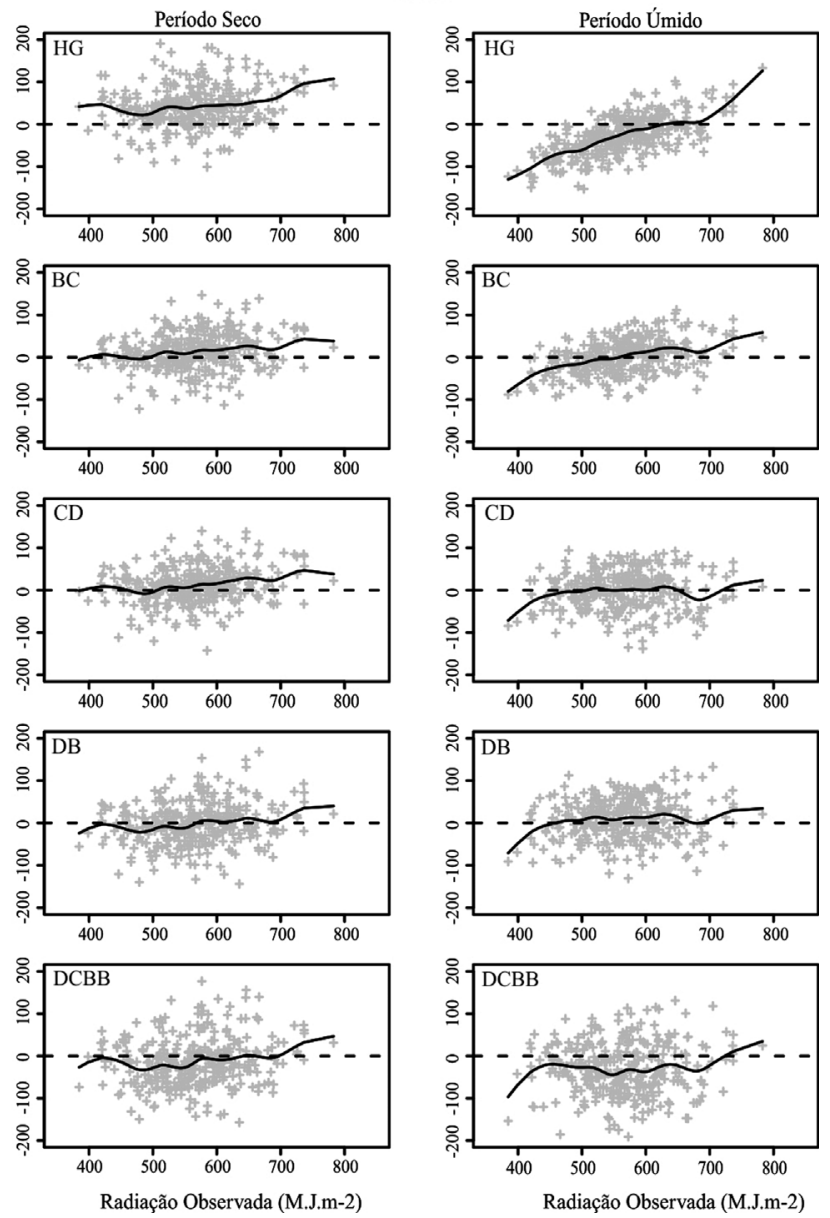

Figura 3 - Desvios da radiação estimada pelos modelos em função dos valores de radiação observada, diárias e mensais, para os períodos seco e úmido e a função "spline" de alisamento cúbica (linha preta) ajustada para os modelos Hargreaves (HG), Bristow-Campbell (BC), Campbell-Donatelli (CD), Donatelli-Bellocchi (DB) e modular DCBB (DCBB). 
20 M.J.m ${ }^{-2}$.dia ${ }^{-1}$ e superestimou para valores acima desse. Esse modelo também apresentou os valores mais baixos para os coeficientes de determinação para ambos os períodos, seco e úmido (Fig. 4). Os modelos BC, CD, DB e DCBB apresentaram desvios médios semelhantes tanto para o período seco e úmido. Os coeficientes de determinação desses modelos variaram de 0,54 (BC) a 0,65 (CD) para o período seco e de $0,63(\mathrm{BC})$ a $0,78(\mathrm{CD}$ e $\mathrm{DCBB})$ para o período úmido.

Para os valores acumulados mensais de radiação solar global, o modelo HG também apresentou o pior desempenho entre os modelos estudados. O modelo HG apresentou o pior desempenho em estimar os valores de radiação solar global para o período úmido. Para o período seco, os modelos $\mathrm{BC}, \mathrm{CD}, \mathrm{DB}$ e DCBB apresentaram menores desvios médios até valores acumulados mensais de 700 M.J.m. ${ }^{-2}$.mês ${ }^{-1}$ (Fig. 3), e apresentaram bons coeficientes de determinação que variaram de $0,69(\mathrm{BC})$ a $0,81(\mathrm{CD})$ (Fig. 4). No período úmido, os modelos $\mathrm{BC}, \mathrm{CD}$ e $\mathrm{DB}$ apresentaram comportamento semelhante ao do período de seca e obtiveram melhores coeficientes de determinação, sendo o valor mais alto de 0,97 (CD) (Fig. 4). Já o modelo DCBB apresentou desvios médios negativos até o limiar de
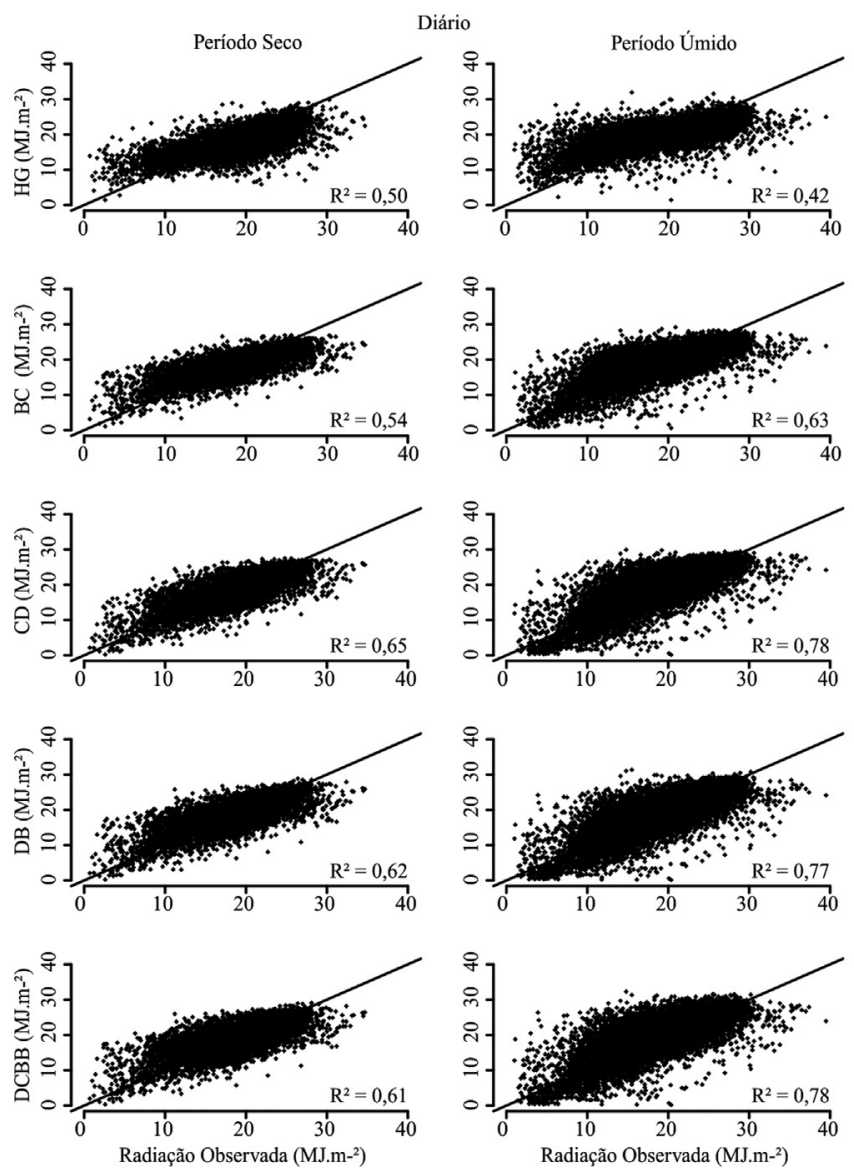

700 M.J.me.-mês ${ }^{-1}$ (Fig. 3) e coeficiente de determinação igual ao do modelo HG (Fig. 4).

A Fig. 5 compara os valores referentes aos índices $\mathrm{de}$ c para os dados diários e acumulados mensais nos períodos seco e úmidos, considerando toda a série histórica. Os modelos apresentaram valores satisfatórios para a concordância (índice d), que variaram de 0,77 (HG) a 0,87 (CD) para os dados diários. Já para a confiança (índice c), o modelo HG mostrou-se o menos preciso. A concordância dos modelos se mostrou maior para os valores de radiação solar acumulados mensalmente, variando entre $0,82(\mathrm{HG}) \mathrm{e}$ 0,91 (CD). A confiança também aumentou, variando de 0,57 (HG) a 0,77 (CD) ("sofrível" a "muito bom" (Camargo e Sentelhas, 1997), respectivamente). Separando os valores simulados em período seco (abril-setembro) e úmido (outubro-março), não foi observado melhoras significativas nos valores de concordância e confiança dos modelos.

\section{Conclusões}

Nos resultados apresentados foram testados cinco modelos de estimativa de radiação solar global (HG, BC, CD, DB e DCBB) para 10 localidades no estado de Goiás, todos tiveram bons índices de concordância levando em conta toda a série histórica de dados, tanto para estimativas
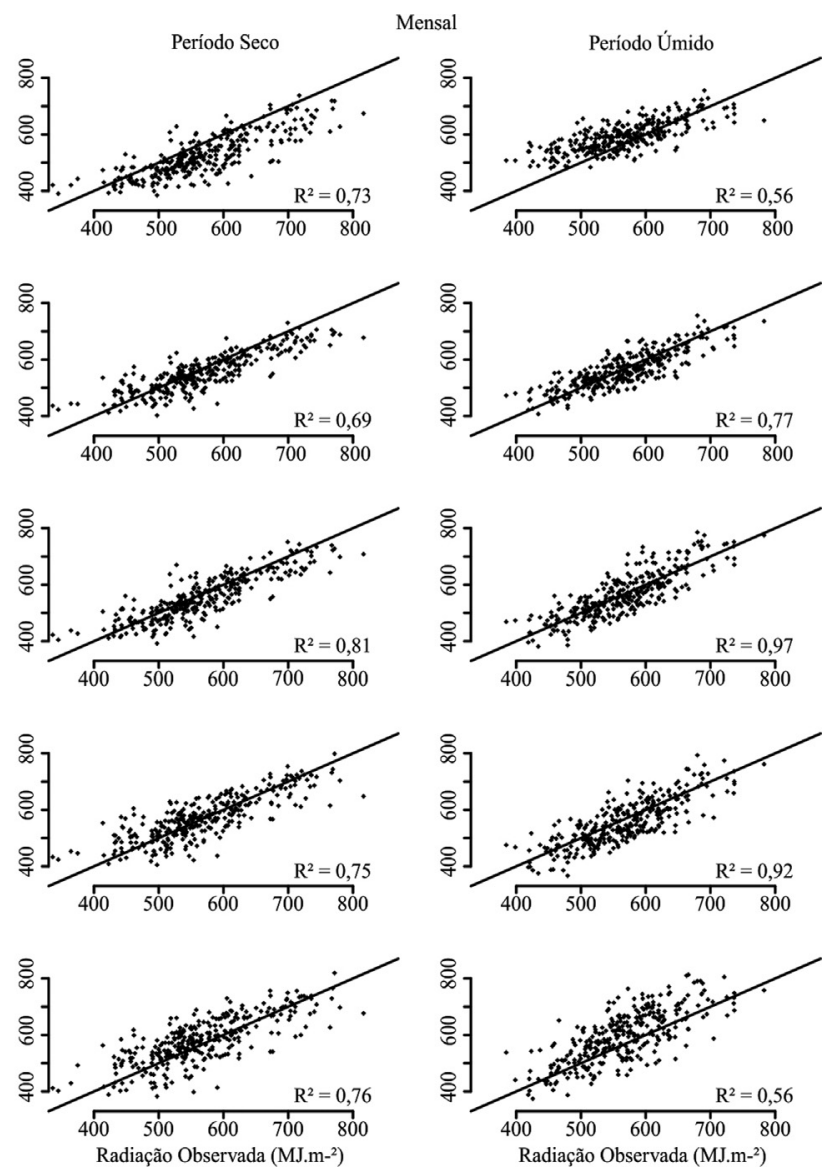

Figura 4 - Regressão entre a radiação solar estimada e observada para os valores diários e mensais de radiação solar para os períodos seco e úmido para os modelos de Hargreaves (HG), Bristow-Campbell (BC), Campbell-Donatelli (CD), Donatelli-Bellocchi (DB) e modular DCBB (DCBB). 

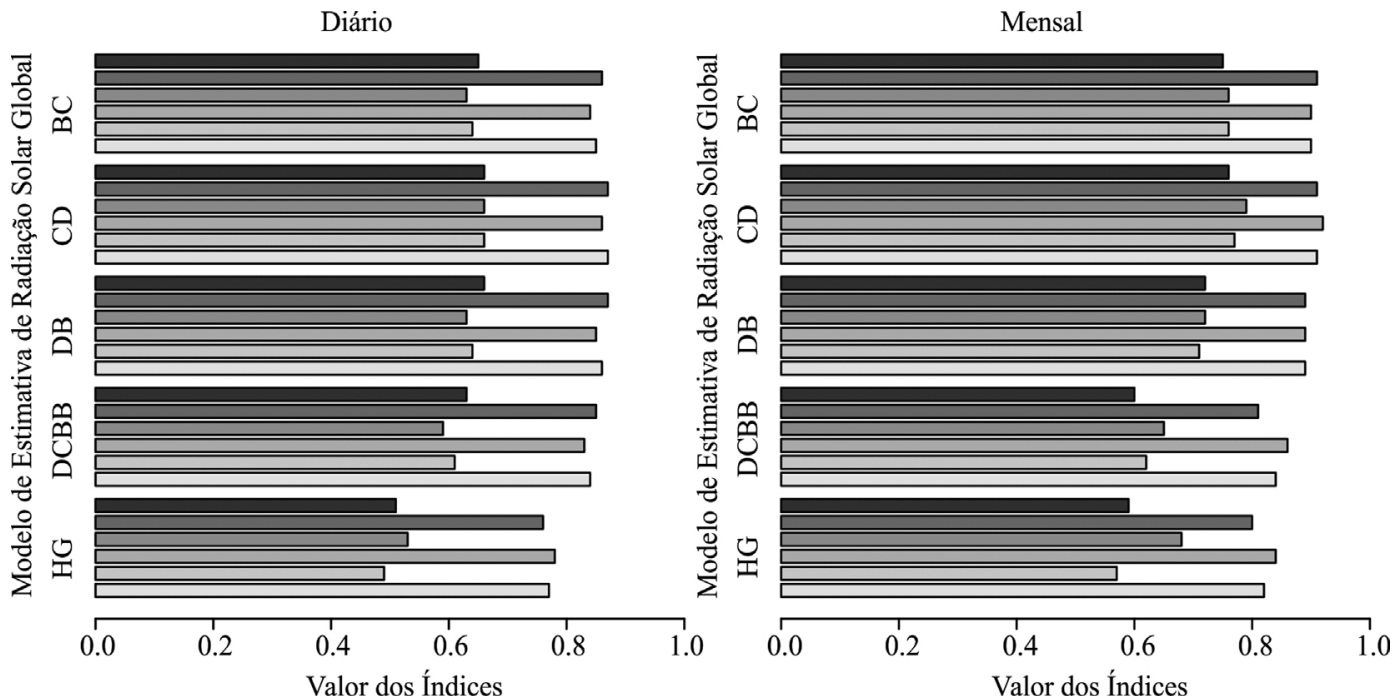

c c-Seco $\square$ c-Úmido $\square$ c - Todo Período

$\square \mathrm{d}$-Seco $\square \mathrm{d}$-Úmido $\square \mathrm{d}$ - Todo Período

Figura 5 - Valores dos índices de concordância (Willmott - d) e confiança (Camargo - c) para valores diários (a) e mensais (b) para toda a série histórica de dados, período seco e úmido.

de radiação solar diárias $(\mathrm{d}=0,77$ a 0,87$)$ quanto mensais ( $d=0,82$ a 0,91). Entretanto, a confiança foi maior nos dados de radiação solar estimada mensalmente $(\mathrm{c}=0,57 \mathrm{a}$ $0,77)$.

Com base nas análises estatísticas, os modelos $\mathrm{BC}$ e DB foram os que apresentaram os melhores desempenhos, sendo que o modelo $\mathrm{BC}$ foi o que obteve os menores ERQM, EQM e EMA. Os modelos DCBB e HG tiveram os piores desempenhos em estimar os valores de radiação solar global para o estado de Goiás. O modelo HG obteve os piores coeficientes de determinação para valores diários e mensais de estimativa de radiação solar $\left(R^{2}=0,41\right.$ e 0,47 , respectivamente) e foi o que mais subestimou/superestimou os valores de radiação solar diária tanto no período seco como no úmido.

\section{Referências}

ABRAHA, M.G.; SAVAGE, M.J. Comparison of estimates of daily solar radiation from air temperature range for application in crop simulations. Agricultural and Forest Meteorology, v. 148, n. 3, p. 401-416, 2008.

BANSOULEH, B.F.; SHARIFI, M.A.; KEULEN, H.V. Sensitivity analysis of performance of crop growth simulation models to daily solar radiation estimation methods in Iran. Energy Conversion and Management, v. 50, n. 11, p. 2826-2836, 2009.

BECHINI, L.; DUCCO, G.; DONATELLI, M.; STEIN, A. Modelling, interpolation and stochastic simulation in space and time of global solar radiation. Agriculture, Ecosystems and Environment, v. 81, n. 1, p. 29-42, 2000.

BRISTOW, K.L.; CAMPBELL, G.S. On the relationship between incoming solar radiation and daily minimum and maximum temperature. Agricultural and Forest Meteorology, v. 31, n. 2, p. 159-166, 1984.
CAMARGO, A.P.; SENTELHAS, P.C. Avaliação do desempenho de diferentes métodos de estimativa da evapotranspiração potencial no Estado de São Paulo. Revista Brasileira de Agrometeorologia, v. 5, n. 1, p. 89-97, 1997.

CARGNIN, A.; SOUZA, M.A. de; CARNEIRO, P.C.S.; SOFIATTI, V. Interação entre genótipos e ambientes e implicações em ganhos com seleção em trigo. Pesquisa Agropecuária Brasileira, v. 41, n. 6, p. 987-993, 2006.

CEBALLOS, J.C. Estimativa de radiação solar à superfície com céu claro: um modelo simplificado. Revista Brasileira de Meteorologia, v. 15, n. 1, p. 113-122, 2000.

CRUZ, C.D.; REGAZZI, A. Modelos biométricos aplicados ao melhoramento genético. Viçosa: UFV, 1997. 390p.

DONATELLI, M.; BELLOCCHI, G. Estimate of daily global solar radiation: new developments in the software RadEst3.00. 2001. In: INTERNATIONAL SYMPOSIUM MODELLING CROPPING SYSTEMS, 2., Florence, 2001. Proceedings. Florence: Institute for Biometeorology, 2001. p. 213-214.

DONATELLI, M.; CAMPBELL, G.S. A simple model to estimate global solar radiation. In: CONGRESS OF EUROPEAN SOCIETY FOR AGRONOMY, 5., 1998, Nitra. Proceedings. Nitra: The Slovak Agriculture University, 1998. v. 2, p. 133-134.

DONATELLI, M.; BELLOCCHI, G.; FONTANA, F. RadEst 3: a software to estimate daily radiation data from commonly available meteorological variables. European Journal of Agronomy, v. 18, n. 3-4, p. 363-367, 2003.

FLETCHER, A.L.; MOOT, D.J. Estimating daily solar radiation in New Zealand using air temperatures. New Zealand Journal of Crop and Horticultural Science, v. 35, n. 1, p. 147157, 2007.

FROELICH, N.J.; SCHMID, H.P. Flow divergence and density flows above and below a deciduous forest. Part II. Below-canopy thermotopographic flows. Agricultural and Forest Meteorology, v. 138, n. 1-4, p. 29-43, 2006. 
GOODIN, D.G.; HUTCHINSON, J.M.S.; VANDERLIP, R.L. Estimating solar irradiance for crop modeling using daily air temperature. Agronomy Journal, v. 91, n. 5, p. 845-851, 1999.

HANSEN, J.W. Stochastic daily solar irradiance for biological modeling applications. Agricultural and Forest Meteorology, v. 94, n. 1, p. 53-63, 1999.

HARGREAVES, G.L.; HARGREAVES, G.H.; RILEY, J.P. Irrigation water requirement for Senegal River Basin. Journal of Irrigation and Drainage Engineering, v. 111, n. 3, p. $265-275,1985$

HEINEMANN, A.B. Caracterização dos padrões de estresse hídrico para a cultura do arroz (ciclo curto e médio) no estado de Goiás e suas consequências para o melhoramento genético. Ciência e Agrotecnologia, v. 34, n. 1, p. 29-36, 2010.

HEINEMANN, A.B.; ANDRADE, C. de L.T.; GOMIDE, R.L.; AMORIM, A. de O.; PAZ, R.L.F. Padrões de deficiência hídrica para a cultura de milho (safra normal e safrinha) no estado de Goiás e suas consequências para o melhoramento genético. Ciência e Agrotecnologia, v. 33, n. 4, p. 10261033, 2009.

HEINEMANN, A.B.; DINGKUHN, M.; LUQUET, D.; COMBRES, J.C.; CHAPMAN, S. Characterization of drought stress environments for upland rice and maize in central Brazil. Euphytica, v. 162, n. 3, p. 395-410, 2008.

HEINEMANN, A.B.; SILVA, S.C.; LOPES JUNIOR, S.; AMORIM, A.O.; ANDRADE, C.L.T.; BASTOS, E.A.; PAZ, R.L.F. Características climáticas dos municípios de Santo Antônio de Goiás (GO), Porangatu (GO), Janaúba (MG), Sete Lagoas (MG), Parnaíba (PI) e Teresina (PI). Santo Antônio de Goiás: Embrapa Arroz e Feijão, 2007. 36p. (Embrapa Arroz e Feijão. Documentos, 214).

HUNT, L.A.; KUCHAR, L.; SWANTON, C.J. Estimation of solar radiation for use in crop modelling. Agricultural and Forest Meteorology, v. 91, n. 3-4, p. 293-300, 1998.

JAGTAP, S.S.; JONES, J.W. Adaptation and evaluation of the CROPGRO-soybean model to predict regional yield and production. Agriculture, Ecosystems and Environment, v. 93, n. 1-3, p. 73-85, 2002.

LIU, D.L.; SCOTT, B.J. Estimation of solar radiation in Australia from rainfall and temperature observations. Agricultural and Forest Meteorology, v. 106, n. 1, p. 41-59, 2001.

LIU, X.; MEI, X.; LI, Y.; WANG, Q.; JENSEN, R.J.; ZHANG, Y.; PORTE, J.R. Evaluation of temperature-based global solar radiation models in China. Agricultural and Forest Meteorology, v. 149, n. 9, p. 1433-1446, 2008.

MAHMOOD, R.; HUBBARD, K.G. Effect of time of temperature observation and estimation of daily solar radiation for the Northern Great Plains, USA. Agronomy Journal, v. 94, n. 4, p. 723-733, 2002.
MAVROMATIS, T.; JAGTAP, S.S. Estimating solar radiation for crop modeling using temperature data from urban and rural stations. Climate Research, v. 29, n. 3, p. 233-243, 2005.

MAVROMATIS, T.; JAGTAP, S.S.; JONES, J.W. El NinoSouthern Oscillation effects on peanut yield and nitrogen leaching. Climate Research, v. 22, n. 2, p. 129-140, 2002.

MCVICAR, T.R.; JUPP, D.L.B. Estimating one-time-of-day meteorological data from standard daily data as inputs to thermal remote sensing based energy balance model. Agricultural and Forest Meteorology, v. 96, n. 4, p. 219-238, 1999.

NASH, J.E.; SUTCLIFF, J. River flow forecasting through conceptual models. Journal of Hydrology, v. 10, n. 3, p. 282290, 1970.

PINKER, R.T.; FROUIN, R.; LI, Z. A review of satellite methods to derive surface shortwave irradiance. Remote Sensing of Environment, v. 51, n. 1, p. 108-124, 1995.

RAMSAY, J.; HECKMAN, N.; SILVERMAN, B. Spline smoothing with model-based penalties. Behavior Research Methods, v. 29, n. 1, p. 99-106, 1997.

REBOITA, M.R.; GAN, M.A.; ROCHA, R.P.; AMBRIZZI, T. Regimes de precipitação na América do Sul: uma revisão bibliográfica. Revista Brasileira de Meteorologia, v. 25, n. 2, p. 185-204, 2010.

TEI, F.; SCAIFE, A.; AIKMAN, D.P. Growth of lettuce, onion, and red beet. 1. growth analysis, light interception, and radiation use efficiency. Annals of Botany, v. 78, n. 5, p. 633643, 1996.

TRNKA, M.; ZALUD, Z.; EITZINGER, J.; DUBROVSKY, M. Global solar radiation in central European lowlands estimated by various empirical formulae. Agricultural and Forest Meteorology, v. 131, n. 1-2, p. 54-76, 2005.

TUNDISI, J.G. Ciclo hidrológico e gerenciamento integrado. Ciência e Cultura, v. 55, n. 4, p. 31-33, 2003.

VALIATI, M.I. Calibração e validação do modelo RadEst 3.0 para estimativa da irradiação solar global em função de medidas de temperaturas do ar máxima e mínima. Botucatu: Universidade Estadual Paulista "Júlio de Mesquita Filho", 2005. 55p. (Tese de Doutorado) - Universidade Estadual Paulista "Júlio de Mesquita Filho", Botucatu, SP.

VENABLES, W.N.; RIPLEY, B. D. Modern applied statistics with S. 4 ed. New York: Springer, 2002. 495p.

WEISS, A.; HAYS, C.J.; HU, Q.; EASTERLING, E.W. Incorporating bias error in calculating solar irradiance: implications for crop yield simulations. Agronomy Journal, v. 93, n. 6, p. 1321-1326, 2001.

WILLMOTT, C.J.; WICKS, D.E. An empirical method for the spatial interpolation of monthly precipitation within California. Physical Geography, v. 1, n. 1, p. 59-73, 1980.

This is an Open Access article distributed under the terms of the Creative Commons Attribution Non-Commercial License which permits unrestricted non-commercial use, distribution, and reproduction in any medium provided the original work is properly cited. 